\title{
Meridianbeobachtungen des Mondes und der Mondsterne in Helsingtors.
}

Die erste Reihe der folgenden Beobachtungen ist im Herbst 1882 von Herrn Professor Sundell am Reichenbach' schen Meridiankreise der hiesigen Sternwarte angestellt worden. Nur an dem einen Abende Oct. 2 I wurde er ron Magister Dreijer vertreten. Mit dem Anfang dieses Herbstes hatte ich mich entschlossen, diese Beobachtungen am Meridiankreise wieder aufzunehmen. Als später Herr Stud. Eklund, der während mehrerer Monate die Zeitbestimmungen in sehr befriedigender Weise besorgt hatte, auf meine Aufforderung sich bereit erklärte, eine ähnliche Beobachtungsreihe auszuführen, uberliess ich ihm den Meridiankreis und habe von October an das Passageninstrument von Utzschneider und Liebherr zu diesem Zwecke benutzt. Die Uhr von Hauth, nach der beobachtet wurde, ist im Meridiansaale in der Nähe des Passageninstrumentes aufgestellt, die Schläge sind aber ohne irgend welche Anstrengung auch dem Beobachter am Meridiankreise gut hörbar. Die Auge- und Ohr-Methode wurde ausschliesslich angewendet.

Gleichzeitig mit den Beobachtungen des Mondes und der Mondsterne wurde vom Anfang October an von beiden Beobachtern eine Zeitbestimmung gemacht, welcher die Stern- positionen des Berliner Jahrbuches zu Grunde gelegt wurden. Die dabei angewendeten Sterne sind in der Columne Zeitstern angefuihrt. Ist dem Namen des Sternes keine weitere Bemerkung hinzugefügt, so wurde der Stern an beiden Instrumenten beobachtet, findet sich aber nach demselben ein D oder ein E, so wurde der Stern nur von mir resp. nur von Herrn Eklund beobachtet. Auch die früheren Beobachtungen beruhen in der Regel auf solchen gleichzeitigen Zeitbestimmungen, im Jahre $\mathbf{r} 882$ unter Benutzung der Ephemeride der 539 Sterne der Astr. Gesellschaft. In Ausnahmefällen wurde die Kenntniss der Zeit in anderer Weise gewonnen, wie jedesmal aus den Columnen "Zeitstern * und , Bemerkungen e hervorgeht.

Ausser dem Mondrande sollten noch die - Moon-Culminating Stars des Nautical Almanac beobachtet werden. Zwei Mal wurde jedoch besonderer Umstände halber wie halb bedeckter Himmel etc. - von dieser Regel abgewichen, indem $\mathrm{r} 883$ Nov. 8 die Mondsterne der Connaissance des Temps beobachtet und ${ }^{2} 883$ Dec. 6 die des Nautical Almanac von I 883 Juli 21 als vorangehende Mondsterne benutzt wurden.

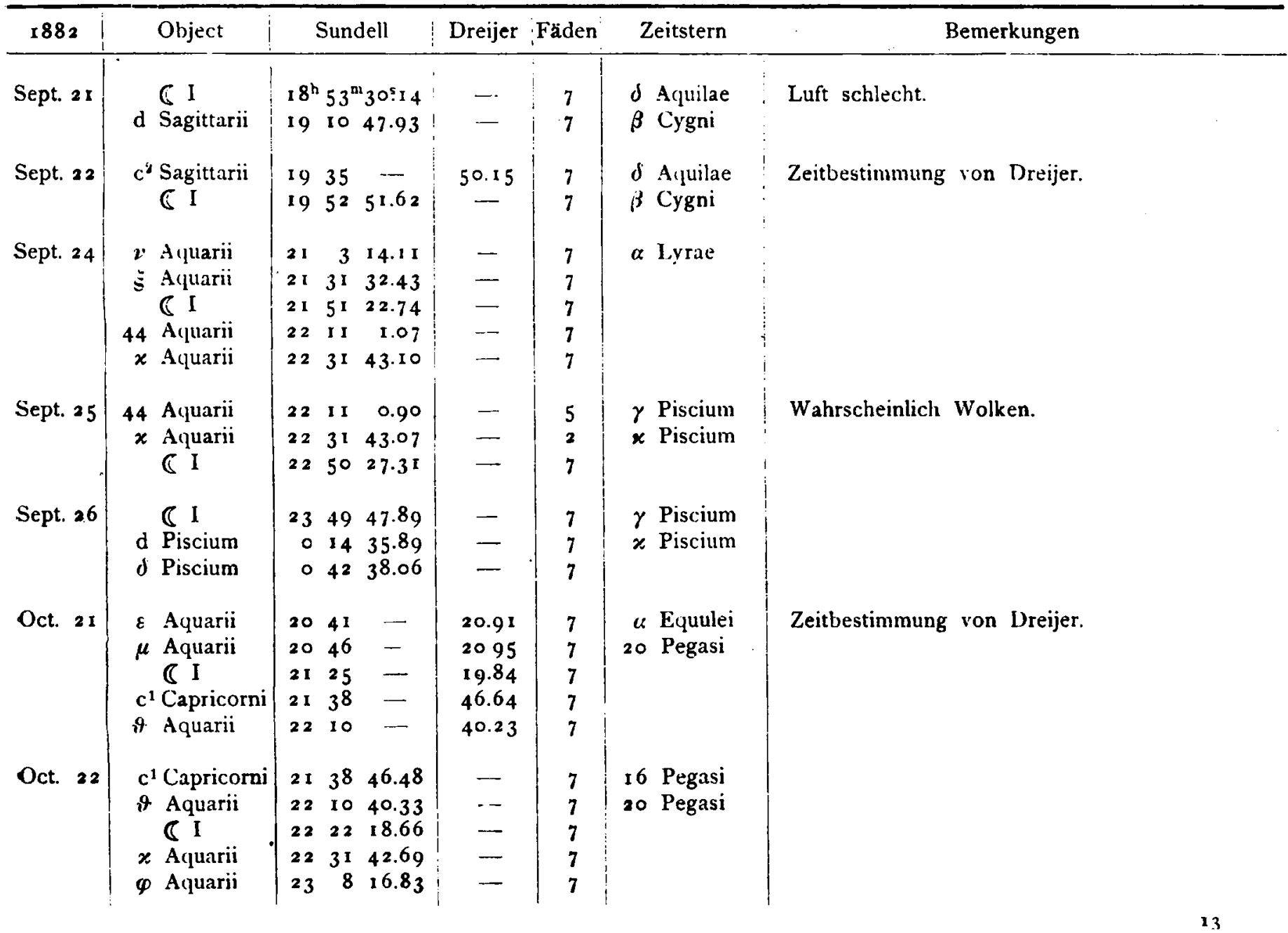




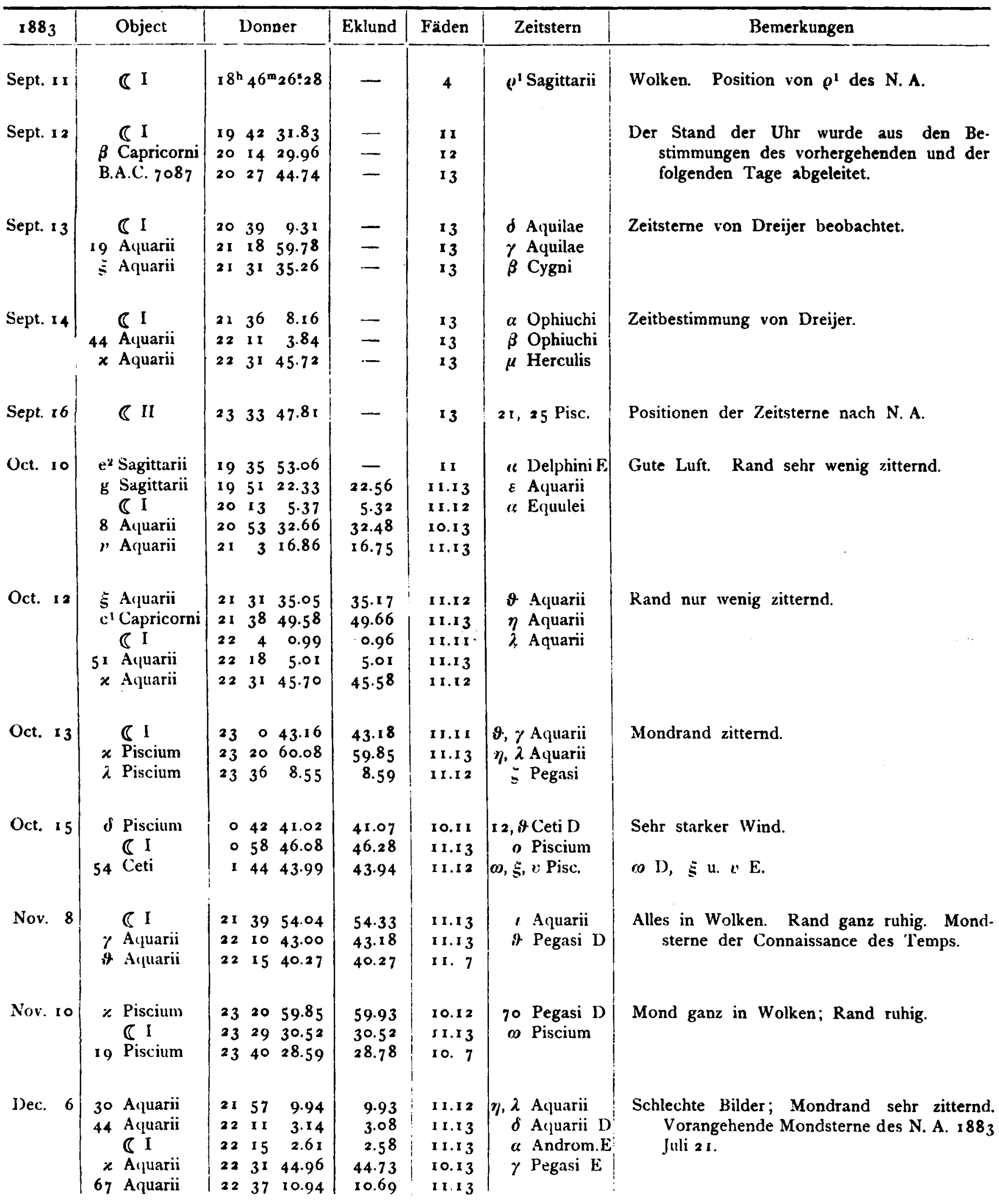




\begin{tabular}{|c|c|c|c|c|c|c|c|c|c|c|c|c|}
\hline \multirow{3}{*}{\multicolumn{2}{|c|}{1882}} & \multirow{3}{*}{\multicolumn{3}{|c|}{ M. Z. Helsingf. }} & \multirow{2}{*}{\multicolumn{3}{|c|}{ AR. $\mathbb{C}$}} & \multicolumn{4}{|c|}{ Correctionen } & \multirow{3}{*}{ Rand } \\
\hline & & & & & & & & \multicolumn{2}{|c|}{ Hansen } & \multicolumn{2}{|c|}{ Newcomb } & \\
\hline & & & & & \multicolumn{2}{|c|}{ Sundell } & Dreijer & S. & Dr. & s. & $\overline{\text { Dr. }}$ & \\
\hline \multicolumn{2}{|c|}{ Sept. 2 I } & \multicolumn{3}{|c|}{$6^{h} 5^{2^{1 n}} 4^{6^{s}} \cdot 25$} & \multicolumn{2}{|c|}{$18^{h} 54^{m} 40: 06$} & - & $-0: 6_{3}$ & - & $+0: 31$ & - & I \\
\hline & 22 & & 48 & 2.18 & 195 & 1.63 & - & $-0.5^{8}$ & - & +0.35 & - & I \\
\hline & 24 & 9 & 38 & 21.75 & 215 & 32.42 & - & -0.83 & 一 & +0.07 & - & I \\
\hline & 25 & 10 & 33 & 20.73 & 225 & $3^{6.98}$ & - & -1.06 & - & -0.17 & - & $\mathbf{I}$ \\
\hline & 26 & & 28 & 35.93 & 235 & 57.82 & 一 & -0.91 & - & -0.02 & - & I \\
\hline \multirow[t]{2}{*}{ Oct. } & 21 & 7 & 26 & 12.41 & 2126 & - & $28: 29$ & - & -0.70 & 一 & $+0: 21$ & I \\
\hline & 22 & 8 & I 9 & 6.03 & 2223 & 27.15 & 一 & -0.78 & - & +0.11 & - & I \\
\hline \multicolumn{2}{|c|}{1883} & \multicolumn{3}{|c|}{ M. Z. Helsingf. } & \multicolumn{2}{|c|}{ Donner } & Eklund & D. & E. & D. & E. & Rand \\
\hline \multirow[t]{5}{*}{ Sept. } & 11 & 7 & 25 & 57.54 & $184 \%$ & 33.74 & - & -0.78 & - & +0.12 & - & I \\
\hline & 12 & 8 & 17 & 58.36 & 1943 & 39.65 & 一 & -0.90 & - & 0.00 & - & I \\
\hline & 13 & 9 & 10 & 30.84 & 2040 & 17.33 & 一 & -0.94 & - & -0.04 & - & I \\
\hline & 14 & 10 & 3 & 24.61 & $2 I 3$ & 16.33 & $\ldots$ & -1.17 & - & -0.27 & $\ldots$ & I \\
\hline & 16 & I I & 50 & $3^{6.49}$ & 233 & 38.93 & - & --1.41 & - & -0.49 & - & II \\
\hline \multirow[t]{4}{*}{ Oct. } & ro & 6 & $5^{8}$ & 20.64 & 201 & I 2.33 & 12.28 & $-0.8 \mathrm{z}$ & -0.87 & +0.08 & +0.03 & I \\
\hline & 12 & 8 & 41 & 6.84 & 22 & 8.62 & 8.39 & -0.98 & $\longrightarrow$ I.2I & -0.09 & $-0.3^{2}$ & I \\
\hline & 13 & 9 & 33 & 44.60 & 23 & 51.45 & $5^{1.47}$ & -1.24 & -1.22 & -0.33 & -0.31 & 1 \\
\hline & 15 & I I & 23 & $3^{8.59}$ & 05 & 56.50 & 56.70 & -1.09 & -0.89 & -0.14 & +0.06 & I \\
\hline \multirow[t]{2}{*}{ Nov. } & 8 & 6 & 30 & 53.22 & 214 & 0.29 & $0.5^{8}$ & -0.97 & -0.68 & -0.07 & +0.22 & I \\
\hline & 10 & 8 & 12 & 31.23 & $233 c$ & 38.22 & 38.21 & -0.94 & -0.95 & -0.01 & -0.02 & I \\
\hline Dec. & 6 & & 15 & 49.73 & 22 re & 8. 18 & 8.15 & -1.06 & -1.09 & -0.18 & $-0.2 \mathrm{I}$ & I \\
\hline
\end{tabular}

Die bei den Operationen zur Erlangung der Daten dieser zweiten Tafel angewendeten Grössen sind der Connaissance des Temps entnomnen. Durch Anbringung des Werthes für die Durchgangszeit des Mondhalbmessers, wie diese in der stiundlichen Ephemeride angegeben ist, wurden die Rectascensionen des Mondmittelpunktes erhalten, welche somit auf den Positionen der Zeitsterne beruhen. Diese Rectascensionen sind alsdann mit denjenigen Werthen verglichen, welche aus derselben Ephemeride mit Hülfe der Columne songitude des lieux où la lune passe au méridienc berechnet wurden, und mit denjenigen, die hieraus durch Anbringung der Newcomb'schen Correction hervorgingen, unter der Annahme, dass die Werthe dieser nach der Tafel der Connaissance für den Pariser Mittag gelten.

$\mathrm{Da}$ nur eine Beobachtung des zweiten Randes vorkommt, schliesse ich diese aus, und finde so die folgenden Fehler als Mittelwerthe aus den Beobachtungen von
Rand I.

Fehler der Mondephemeride nach Anbringung dér Newcomb'schen Correction aus den obigen Beobachtungen abgeleitet:

\begin{tabular}{|c|c|c|c|}
\hline & S. u. Dr. & D. & E. \\
\hline $\begin{array}{l}\text { Anzahl der Beob. } \\
\text { Beob. Corr. im Mittel } \\
\text { Wahrsch. Fehler }\end{array}$ & $\begin{array}{c}7 \\
+0 ! 123 \\
\pm 0.046\end{array}$ & $\begin{array}{c}11 \\
-0.085 \\
\pm 0.027\end{array}$ & $\begin{array}{c}7 \\
-0.079 \\
\pm 0.052\end{array}$ \\
\hline
\end{tabular}

Die noch ubrig bleibenden Abweichungen von diesen Werthen lassen einen Fehler von kurzer Periode kaum verkennen; die Beobachtungen sind jedoch zu wenig zahlreich, um darauf Schluisse zu bauen.

Helsingfors 1884 Febr. 6.

Anders Donner.

Sternbedeckungen und Jupitersmonde, beobachtet zu Gohlis bei Leipzig. $\varphi=51^{\circ} 2 I^{\prime} 35^{\circ} \circ \quad \lambda=49^{\mathrm{m}} 29^{\mathrm{m}} 6{ }_{5}$ östl. von Greenw.

Die Beobachtungen sind mit einem Steinheil'schen Refractor von $108^{\mathrm{mm}}$ Oeffnung mit 120 facher Vergrösserung gemacht, nur bei der Sternbedeckung ain 3. März I88 I wurde ein Fernrohr von $68^{\mathrm{min}}$ Oeffnung mit 90 facher Vergrösserung angewandt. Die angegebene Zeit ist mittlere Ortszeit, die Zeitbestimmungen geschahen in der Regel im unmittelbaren Anschluss an die Beobachtungen, nur bei einigen
Beobachtungen der Jupitersmonde wurde der Uhrstand interpolirt.

Die Erscheinungen der Jupitersmonde sind mit denselben Abkürzungen bezeichnet, wie in Nautical Almanac, mit dessen Ephemeride auch die Beobachtungen verglichen sind. Es ist stets der Moment des ersten resp. letzten Lichtschimmers notirt. 